\title{
La gamificación en el aula E/LE multigeneracional: la «habitación de escape» como modelo de propuesta didáctica
}

\author{
María PALANCA Climente \\ Hispania, escuela de español \\ maria.palanca@hispania-valencia.com \\ Alicia Ramos CASTILlO \\ Hispania, escuela de español \\ alicia.ramos@hispania-valencia.com
}

Resumen: En este artículo se ofrece un enfoque para integrar en el aula de E/LE diversas competencias generales y destrezas lingüísticas a través de la gamificación que incentiva la competencia comunicativa del estudiante. Este enfoque nace de la reflexión sobre la necesidad de incorporar nuevas estrategias de enseñanza basadas no solo en lo cognitivo sino que mezcle también el componente emocional. La Neuroeducación nos acerca a la gamificación como medio para motivar y despertar emociones que faciliten el proceso de aprendizaje. La emoción y la cognición van unidas y se interrelacionan entre sí para dar como resultado la conducta del estudiante. El objetivo es que el estudiante participe en una «habitación de escape» y para lograr la clave final deberá resolver diversas pruebas en las que trabajará gramática, comprensión auditiva, lectura y adquisición de léxico. Todo estará guiado a través de un componente cultural común: la literatura española y latinoamericana.

Palabras clave: gamificación, Neuroeducación, nuevas estrategias de enseñanza, habitación de escape.

\section{Gamification in the multigenerational E/LE classroom: the "escape room" as a model of a didactic proposal.}

\begin{abstract}
Included in this article is a new insight to integrate several core competences and linguistic skills in the $E / L E$ classroom. This approach is displayed through gamification, which incentives the student communicative skills. It emerges from the consideration of the need to incorporate new learning strategies based, not only on the cognitive component, but also the emotional. Neuroeducation brings gamification as a way to motivate and trigger emotions which facilitates the learning process. Both emotion and cognition are integrated processes that work as one, in order to give the student's conduct as the final outcome. The aim of the proposal is to achieve the participation of the student in an "escape room", in which the goal is a quest to solve several tasks in order to obtain a final clue. Throughout the process grammar, lexicon, listening and reading skills are developed. Spanish and South American literature are the common cultural components guiding the whole learning experience.
\end{abstract}

Keywords: gamification, Neuroeducation, new learning strategies, escape room. 


\section{Introducción}

El presente artículo nace desde la reflexión sobre la evolución generacional que convive en el aula E/LE y con ella sobre la necesidad de incorporar estrategias de enseñanza que se adapten al momento. El siglo XXI nos regala una oportunidad maravillosa de convivencia entre diversas generaciones que cuentan con marcas de personalidad muy diferentes y esto es con lo que lidiamos los profesores de español como segunda lengua (L2 a partir de ahora).

Según la relación con la tecnología y el mundo laboral, y basándonos en las conclusiones del Diagnóstico de la Diversidad Generacional realizado por el Observatorio Generación \& Talento, con la Universidad de Pontificia Comillas, podemos hablar de unas cuatro generaciones:

1. Los Baby Boomers (nacidos entre 1945 y 1964). Esta generación entiende el trabajo como algo estable, a largo plazo adictivo y no es prioritario para ellos que su trabajo sea vocacional. No le dedican mucho tiempo al ocio y entre ellos persiste generalmente el ideal de familia tradicional, aunque poco a poco se están rompiendo estructuras.

2. La Generación X (nacida entre 1965 y 1981). Son los que vieron el nacimiento de Internet y los avances tecnológicos. Están marcados por grandes cambios sociales, una generación en transición a la que también se le llamó Generación Perdida o Peter Pan. Logran mantener de manera equilibrada la relación entre la tecnología y la vida social activa «presencial». Aceptan las órdenes de la jerarquía institucional, es decir que suelen ser menos emprendedores y viven cómodos desde la figura del empleado. Son los padres de los Millennials y viven adaptándose a la nueva generación.

3. La Generación Y o Millennials (nacidos entre 1982 y 1994). Viven adaptados completamente a la tecnología, pero todavía conservan algunos códigos de privacidad en relación a lo que exponen o no en Internet. Son multitasking, pero no workaholic. Suelen ser creativos, idealistas, emprendedores, aman viajar, conocer mundo y subir fotos a las redes. Para ellos, la emoción es una de las claves de la comunicación.

4. La Generación Z o Centennials (nacidos desde 1995 hasta el presente). Son auténticos «nativos digitales», desde niños usan Internet. Sus mejores profesores son los tutoriales. Hablamos de una generación altamente creativa y de alguna manera sobreinformada, lo que les lleva en ocasiones a sentirse en posesión de la verdad absoluta. Comparten en las redes sociales su vida privada y su vida social pasa un alto porcentaje por las redes. Miden su éxito a través de «compartidos» y «likes» y se comunican con símbolos e imágenes. Son comunicadores ágiles pero poco precisos, lo que lleva a sus mensajes a un amplio margen de interpretación. Son más pragmáticos que los Millennials, buscan innovar con lo que tienen a su alcance. Les preocupa encontrar una vocación acorde a sus gustos, conocerse a sí mismos y aceptar las diferencias en un mundo cada vez más globalizado. Les gusta colaborar y hablan de valor, no de precio. Necesitan sentir curiosidad, les gusta pensar y aspiran a ser expertos. 


\subsection{Cada época lo suyo: diferentes necesidades en el proceso de enseñanza- aprendizaje.}

El paso de una generación a otra denota cambios de necesidades en todos los ámbitos. Vivimos renovaciones en la manera de comunicarnos, reorganizaciones en la manera de relacionarnos, necesitamos cosas diferentes para alcanzar una sensación de plenitud y bienestar. En menos de 80 años se han creado barreras en ocasiones infranqueables si no somos conscientes de ellas.

Estos cambios no escapan a las necesidades que surgen en el aula E/LE. El aprendizaje de lenguas lleva consigo también una evolución en la manera de conectar y desarrollar las diversas habilidades, destrezas lingüísticas y comunicativas.

¿Cuáles son las claves para facilitar aprendizajes adaptados al escenario actual? Podemos basarnos en algunos principios que presenta la Neuroeducación. Como nos comenta Mora, F. (2013: 98) en su libro Neuroeducación, «esta es una nueva disciplina en la que conocimientos generados por la neurociencia, la educación y la psicología confluyen y juntos nos pueden aportar información significativa sobre el proceso de enseñanza y aprendizaje».

La Neuroeducación se basa en el principio de que la emoción y la cognición son procesos que van unidos y que se interrelacionan entre sí para dar como resultado final la conducta del sujeto. Hablaremos pues de un aprendizaje significativo. En el proceso de aprendizaje de un sujeto lo que abre la puerta a aprender es la emoción. Esta es la que despierta en las personas la curiosidad, el interés y por tanto el foco de la atención.

La atención nace de algo que puede significar recompensa (placer) o castigo (peligro) y que por tanto tiene que ver con la supervivencia del individuo. Atendemos a aquello que nos interesa porque nos proporciona una recompensa positiva o porque nos evita una recompensa negativa. Aquello que no resulta significativo para nosotros, no suscita nuestra atención y solo con ella llega el aprendizaje. En lo relativo al aprendizaje, sabemos que este va unido a las emociones y, si bien no nos han enseñado a reconocer y gestionar las emociones, de alguna manera es necesario que esto cambie, pues las emociones son importantes en la educación. Como dice Damasio, A. (2005: 9) «no somos máquinas pensantes que sentimos, somos máquinas sentimentales que pensamos».

Ahora bien, ¿cómo suscitar la atención entre estas últimas generaciones caracterizadas por tener un acceso rápido y directo a la información? Estas generaciones nacidas lejos del mundo analógico del papel y el libro, de la jerarquía del profesor maestro y de la obligación y el esfuerzo como norma de aprendizaje son generaciones que para nosotros como profesores de español son un público ante el que tenemos ganada la voluntad de aprender una lengua, puesto que son generaciones globalizadas que cuentan entre sus valores con la necesidad de comunicación en diferentes lenguas.

No obstante, el reto está en llegar a su cognición para que les resulte interesante y significativa la manera en la que se les muestra el conocimiento. Por eso, ante generaciones que necesitan sentir y vivir lo que aprenden desde la colaboración y la experiencia, contamos con recursos rescatados del contexto laboral y traídos al ámbito docente gracias a estudios neuroeducativos.

Si tenemos en cuenta que un gran grupo de docentes de español como L2 forman parte de la Generación X o son Millennials y que un gran porcentaje de estudiantes de español como lengua extranjera están dentro de la Generación Y o son Centennials, entenderemos 
cómo conceptos generalizados ya en la didáctica como es el de la gamificación entra pisando fuerte también en el mundo de E/LE puesto que la emoción y el autorrefuerzo es uno de los elementos clave tanto en las necesidades de las nuevas generaciones como en los recursos aplicados en la gamificación.

\section{2 ¿De qué hablamos cuando hablamos de gamificación?}

Está de moda utilizar términos que definen conceptos con los que hemos convivido siempre, pero que no habíamos acuñado con un término concreto. Esto es lo que ha sucedido, en cierto modo, con la definición de la gamificación. Como nos presentan Foncubierta, J.M. y Rodríguez, C. (2014) en su artículo Didáctica de la gamificación en la clase de español, la gamificación (ludificación, si no usamos el extranjerismo) se define como «el empleo de elementos y del pensamiento del juego en contextos de no juego». Este término no nace acuñado al contexto de la educación sino que surge en ámbitos laborales o de Recursos Humanos. No obstante, ¿qué profesor no ha utilizado el marco del juego en el aula para dinamizar alguna actividad? ¿Quién no ha optado por utilizar el recurso de dar puntos a los estudiantes que dan con la respuesta correcta o son más rápidos, o de crear equipos para competir entre los estudiantes a modo de motivación? No estamos hablando de nada nuevo exclusivo de las nuevas estrategias de aprendizaje, también se utilizaban elementos lúdicos en el aula en la era analógica.

Aplicado al aula E/LE el concepto de gamificación podría adaptarse a la definición que se da en el artículo nombrado anteriormente: J.M Foncubierta y C. Rodríguez dan en su artículo:

Técnica que el profesor emplea en el diseño de una actividad de aprendizaje (sea analógica o digital) introduciendo elementos del juego (insignias, límite de tiempo, puntuaciones, dados, etc.) con el fin de enriquecer esa experiencia de aprendizaje, dirigir y/o modificar el comportamiento de los alumnos en el aula.

Foncubierta, J.M. y Rodríguez, C. (2014: 2)

En otras palabras, gamificar es conseguir que se aprenda aquello que inicialmente no motiva mediante elementos del juego. Gamificar es fomentar el aprendizaje activo. La novedad y lo inesperado son los principales nutrientes para nuestro cerebro y a las nuevas generaciones nos encantan las sorpresas. Cuando llevamos la gamificación al aula se crean escenarios que estimulan la creatividad, las ganas de colaborar, de criticar, de analizar, de aprender.

Los elementos que podemos utilizar para gamificar son, por ejemplo, dinámicas basadas en emociones, narrativas que descubrir, historias en progresión o elementos conectados. Encontramos mecánicas de gamificación como los desafíos o los retos incluso la cooperación en el grupo. Los componentes más comunes que encontraremos serán los nombrados puntos, los bonus o incluso los avatares.

Es aquí donde conecta la importancia de encontrarnos en el aula ante un tipo de generación o de otra, bien sea en el papel del docente como en el del estudiante. Al contar en este ámbito con generaciones digitales, creativas, con carácter colaborativo, de atención cada vez más diversificada y a las que les gusta tener control y preferencia tenemos en este nuevo recurso un aliado perfecto. 
En conclusión, la gamificación en el aula de E/LE surge de las nuevas maneras de comunicarnos. Si hay cambios en los estudiantes y en los profesores, habrá cambios en el aula. Gracias a elementos del juego se logra conectar con una nueva generación de estudiantes que refleja el cambio social. Así, llevamos los nuevos elementos que rigen la comunicación social al aula para despertar la motivación de estudiantes acostumbrados a las multitareas, a la rapidez, la recompensa, la búsqueda del valor, las pantallas.

\section{La «habitación de escape» como propuesta didáctica: «La entrevista más emocionante de mi vida».}

«Lo escuché y lo olvidé. Lo vi y lo entendí. Lo hice y lo aprendí», esta cita de Confucio refleja uno de los principios más importantes de la Neuroeducación, pues es en la práctica donde el proceso cognitivo conecta más directamente con la emoción que nos hará recordar lo vivido como parte del aprendizaje. Si experimentar es la clave de la didáctica, cuánto más lo será en el aula E/LE, laboratorio como escenario, en ocasiones casi único, donde practicar la lengua que se está aprendiendo.

Nuestra propuesta didáctica es una «habitación de escape». Como profesoras nos convertimos en guías que ayudan a los estudiantes a entender y a practicar las destrezas necesarias para mejorar sus habilidades lingüísticas. No obstante, lo interesante en esta sesión es que los estudiantes deberán ser capaces de aplicar y demostrar diferentes reglas, procedimientos y conocimientos que han ido trabajando a lo largo de su curso para poder ayudar a escapar al protagonista de la historia de una habitación en la que se encuentra encerrado.

Con este modelo de gamificación pretendemos sacar a los estudiantes de la espiral de las clases más convencionales en las que la pizarra y las actividades más tradicionales los hacen meros receptores de la información. Con ello, buscamos convertirlos en colaboradores responsables y activos de su aprendizaje. Su implicación y su participación serán las herramientas más directas para su propia evolución.

La actividad está pensada para estudiantes de nivel B2 según los Niveles Comunes de Referencia del MCER y está secuenciada para una sesión de 2 horas. Las dinámicas buscan el trabajo colaborativo por lo que siempre se desarrollarán bien en parejas, en equipo o en gran grupo. En esta actividad planteamos el trabajo de diversas destrezas y habilidades lingüísticas a través de un recorrido de pruebas con diferentes formatos.

Hemos recreado la historia de Mario, un personaje a través del cual en cualquier aula podrá llevarse a cabo esta actividad sin demasiada complicación. El hecho de que no sea el estudiante el que deba escapar, sino el personaje de ficción facilita la adaptación de la actividad a diversos contextos.

¿Qué necesitamos para llevar a cabo esta «habitación de escape»?

1. Un proyector o pantalla para el visionado de las grabaciones.

2. Dispositivo electrónico como teléfonos móviles, tabletas o Ipads.

3. Acceso a Internet para la reproducción de vídeos en páginas como YouTube.

4. Lector de código QR o acceso a alguna aplicación para descargarlo.

5. Tarjetas y material fotocopiable que facilitará el profesor con las instrucciones de cada prueba.

6. Energía y muchas ganas de aprender y pasarlo bien. 


\subsection{Introducción a la actividad. La entrevista de trabajo más emocionante de mi vida.}

Mario es un joven estudiante de Filología amante de la literatura al que han llamado para una entrevista de trabajo en una de las librerías más antiguas de la ciudad de Valencia. Está emocionado por la oportunidad que le brindan, pero la aventura comienza cuando al llegar a su cita, casi sin darse cuenta, se encuentra encerrado en la trastienda de la librería. Mario necesitará de la ayuda de los estudiantes para poder salir airoso de la trastienda y con ello, de la entrevista de trabajo más emocionante de su vida.

\subsection{Actividad contextualizadora. La ilusión de Mario.}

Esta actividad comienza trabajando una de las destrezas lingüísticas receptivas con una audición en la que un narrador sitúa al estudiante en la historia. En ella el estudiante tendrá que estar muy atento para entender qué está pasando y poder responder a unas preguntas básicas (ficha 1 de material para el profesor) que evaluarán la comprensión de la tarea.

Material: ficha 1, audio 1.

\subsection{Prueba 1. Artes de librero.}

Esta actividad se centra en la adquisición de léxico a través de la comprensión lectora. Contamos con competencias transversales como sus habilidades para el trabajo organizativo en reparto de tareas dentro el grupo y los recursos cognitivos con los que activar el léxico adquirido. De esta manera empezamos con un enfoque colaborativo que conecta con uno de los valores principales para la motivación de la "generación de la última generación".

El profesor facilita a los estudiantes la nota que Mario recibe al empezar su entrevista de trabajo. En ella aparecerán las instrucciones para realizar la primera prueba. En esta los estudiantes deben unir sustantivos con sus definiciones y todo ello lo encontrarán en tarjetas. El campo léxico trabajado es el mundo de los libros, que acercará a Mario a las habilidades que son imprescindibles para ser un buen librero. La actividad se realizará en gran grupo de manera que los estudiantes se moverán por el aula buscando la tarjeta que sea su pareja. Al unir las dos tarjetas descubrirán en el dorso una letra. Uniendo todas las letras conseguidas de las parejas llegarán a la palabra clave que permitirá a Mario encender el ordenador de la trastienda a través del cual podrá comunicarse con ellos en persona.

Material: tarjetas de léxico.

\subsection{Prueba 2. ¡Hola, chicos! ¿Estáis ahí?}

Tras un vídeo en el que por fin le ponen cara a Mario entramos en la competencia gramatical. Aquí entran en juego las destrezas lingüísticas tanto productivas como receptivas, pues ellos deberán completar con el modo adecuado las opiniones escritas que encontrará Mario sobre un famoso libro de la literatura española (ficha 2) 
Si completan correctamente los huecos descubrirán una palabra que los llevará a la siguiente pista. El objetivo es encontrar el título del famoso libro del que hablan las opiniones de los lectores.

Material: vídeo 1 , ficha 2.

\subsection{Prueba 3. ¿Dónde estamos? La ciudad escondida.}

En esta actividad hacemos más hincapié en la competencia cultural, puesto que a través de un vídeo reconocerán la ciudad escenario de diversas novelas de la literatura española.

Ayudarán a Mario a elegir la novela correcta a través de un juego de votaciones tras el visionado del vídeo al que accederán mediante un código QR que han conseguido en la prueba anterior.

Material: vídeo 2, código QR, vídeo 3, tarjetas de portadas de libros propuestos.

\subsection{Prueba 4. Ojo al dato: une las piezas clave.}

Mario está muy contento porque gracias a la ayuda de los estudiantes conseguirá con éxito alcanzar su objetivo: trabajar rodeado de libros.

En esta prueba volvemos a dar prioridad a la adquisición de léxico, pero en esta ocasión es importante el cambio de registro. A través de puzles en los que unirán una expresión coloquial, la imagen que la define y un ejemplo de uso descubrirán una palabra que hace referencia al título del siguiente libro.

Material: vídeo 4, tarjetas puzle.

\subsection{Prueba 5. EI orden de la trastienda.}

Nos acercamos al final de la actividad y en esta prueba trabajaremos las destrezas lingüísticas tanto productivas como receptivas. A través de oraciones desordenadas deberán dar con la definición de tres palabras. Estas palabras forman el último título que Mario debe buscar para salir airoso de la trastienda.

Material: vídeo 5, ficha 3.

\subsection{Prueba 6. Ya lo tienes, ¡vocaliza!}

Llegar a la clave final es muy sencillo. Los estudiantes ya tienen los títulos de los 4 libros que nos han ayudado a llegar hasta aquí. Esta prueba será rápida y solo deberán contar las vocales que aparecen en los títulos. Conseguirán así la clave numérica que hará que Mario pueda abrir la puerta.

En esta ocasión contamos con sus competencias transversales y sus habilidades cognitivas para solucionar el reto. Seguro que es un éxito.

Material: vídeo 6, ficha 4 y tarjetas de las portadas de los libros. 


\section{Conclusión.}

El componente más cultural de la propuesta didáctica es el contexto ambientado en el ámbito de la literatura española y latinoamericana. En la sesión de trabajo propuesta presentamos dinámicas para trabajar las diferentes competencias y destrezas necesarias en la evolución del estudiante, pero también hemos intentado hacer un guiño a los docentes.

Hay una viñeta al más genuino estilo de Forges en la que a partir de una idea de L. Miquel y del texto de N. Alarcón se bromea sobre la polivalencia del profesor de español como lengua extranjera. Como comentábamos en el apartado 1 de este trabajo, en el aula E/LE en ocasiones conviven varias generaciones de alumnos, pero uno de los puntos más interesantes es la convivencia generacional dada entre los docentes y los estudiantes. Como docentes no podemos quedarnos aislados en una única y absoluta visión de la enseñanza. Por eso, esta viñeta es tan significativa en cuanto a nuestra función de enseñantes.

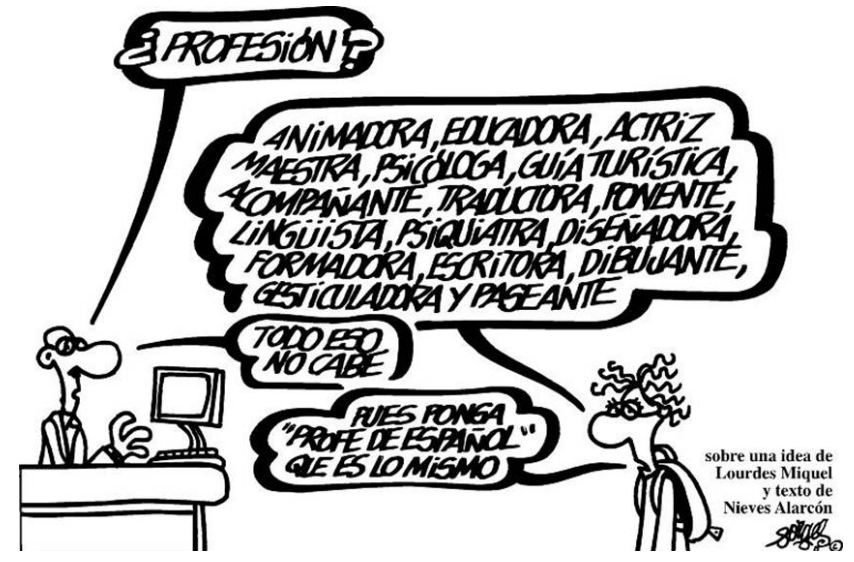

Imagen 1. Viñeta inspirada en el estilo de Forges que bromea sobre la profesión de "profe de español».

A través de los personajes literarios que protagonizan las novelas a las que nos llevaban las pruebas de la habitación de escape podemos reflexionar sobre los valores y las actitudes que el docente de español como L2 necesita transmitir en el aula para contagiar pasión por el aprendizaje y motivar a los estudiantes.

El Ingenioso Hidalgo Don Quijote de la Mancha de Miguel de Cervantes, Sin noticias de Gurb de Eduardo Mendoza; Manolito Gafotas de Elvira Lindo y Cien años de soledad de Gabriel García Márquez. En ellos Mario encuentra la información necesaria para poder convertirse en un auténtico librero independiente y preparado para salir de la trastienda y compartir su amor por la literatura.

¿Qué podemos aprender de ellos para nuestra función de profesores de español? Don Quijote representa la locura y las ganas de emprender nuevos retos. En ocasiones es necesario luchar contra molinos que se ponen en nuestro camino, pero seguimos luchando por nuestros ideales y objetivos.

Ese extraterrestre que se adentra en la jungla de Barcelona en busca de su amigo Gurb que bajo la apariencia física de Marta Sánchez se ha perdido en la ciudad nos descubre uno de los valores más frescos con los que debe contar un profesor: la curiosidad y las 
ganas de sorprenderse. El docente de E/LE vive en al servicio de la multiculturalidad y el viejo refrán de "nunca te acostarás sin saber una cosa más" hace honor a lo que vivimos en el aula.

Manolito Gafotas es claro y directo, sincero y analítico. No juzga, describe. Este talento que se les atribuye a los niños es muy importante para poder entablar una relación de confianza con el estudiante y poder trabajar como guía en el camino del aprendizaje.

Quizás en los Buendía no encontramos un valor relevante que conectar con el profesor de E/LE, la soledad no es la marca de esta profesión, más bien lo contrario, para ser profesor debe gustarte la gente y debes disfrutar compartiendo con ella cada día. Es mucho más interesante quedarnos con la convivencia entre lo maravilloso y lo cotidiano que se da en Macondo. Allí es capaz de vivirse lo inverosímil. Esta idea sí que la trasladamos al aula E/LE, no desperdiciemos la oportunidad de crear pequeños Macondos en nuestras clases. Juguemos, inventemos, soñemos, creemos situaciones imaginarias que nos acerquen a la realidad que vivirán nuestros estudiantes en los contextos en los que necesitarán usar el español como lengua vehicular.

Soñador, emprendedor, curioso, sincero, claro, facilitador de nuevas posibilidades, estos son algunos de los adjetivos con los que podríamos ampliar la viñeta de Forges para definir la figura del profesor en el aula. Y si bien nos fijamos, todos estos valores conectan directamente con emociones, las mismas que moverán a nuestros estudiantes a motivarse por seguir las clases de manera activa, dinámica y enriquecedora. No olvidemos conectar en clase algo más que las pantallas de nuestros estudiantes.

\section{Bibliografía}

Aguilar, I. (2017). «El futuro de la comunicación política: emociones y Generación Z». Revista de Estudios de Juventud, 114, 59-70.

Aguilar, I. (2015). Emociones colectivas: La imprescindible sensibilidad de la política. Recuperado el 21/11/2017, de: http://www.eldiario.es/tribunaabierta/ Emocionescolectivas-imprescindible-sensibilidad-politica_6_446965308.html.

AmEs, C. (1990). «Motivation: What Teachers Need to Know». Teachers College Record, 91 (3), 409-421.

Blakemore, S. y Frith, U. (2011). Cómo aprende el cerebro, las claves para la educación. Barcelona, Ariel.

Cortizo, J.C., Carrero, F., Monsalve, B., Velasco,A., Díaz del Dedo, L. I., \& Pérez MARTín, J. (2011). «Gamificación y Docencia: Lo que la Universidad tiene que aprender de los Videojuegos». En VIII Jornadas Internacionales de Innovación Universitaria. Retos y oportunidades del desarrollo de los nuevos títulos en educación superior. Recuperado el 12/1/2018, de: http://www.aulavirtual.urjc.es/moodle/pluginfile.php/ 2103055/mod_resource/content/1/46_gamificacion.pdf

DAMASIO, A. (2005). En busca de la neurobiología de la emoción y los sentimientos. Barcelona: Editorial Grupo Planeta.

ESTAIRE, S. (2009). El aprendizaje de lenguas mediante tareas: de la programación al aula. Madrid: Editorial Edinumen. 
FERNÁNDEZ-PEQUEÑo JM, J. UlLOA-Hung (2006). «¿Profesores ciegos o estudiantes invisibles?: Incomunicación y brecha generacional». Tendencia \& Retos, 11, 189-201. Recuperado de: http://www.ts.ucr.ac.cr/binarios/pela/pl-000281.pdf

FonCUBIERTA, J.M. \& RodríGUEZ, (2014). Didáctica de la gamificación en la clase de español. Recuperado el 20/1/2018, de: http://www.edinumen.es/spanish_challenge/ gamificacion_didactica.pdf

Herrera, F., J.T. Pujolá y V. CASTRILlejo (17 de noviembre de 2014). L deLengua 86 y la gamificación en el aula de segundas lenguas [audio en podcast]. Recuperado el 13/12/2017, de http://eledelengua.com/gamificacion-aula-segundas-lenguas/

InTITUTO Cervantes (2006). Plan curricular del Instituto Cervantes. Niveles de referencia para el español. Recuperado el 07/04/2017, de: http://cvc.cervantes.es/ensenanza/biblioteca_ele/plan_curricular/

Johnson, S. (2011). Cultura basura, cerebros privilegiados. Barcelona: Roca Editorial.

JENSEN, E. (2004) Cerebro y aprendizaje: competencias e implicaciones educativas. Madrid: Narcea S.A. Ediciones.

MORA, F. (2013). Neuroeducación: sólo se puede aprender aquello que se ama. Madrid: Alianza Editorial. 